\title{
Propagation of whistler-mode chorus to low altitudes: divergent ray trajectories and ground accessibility
}

\author{
J. Chum ${ }^{1}$ and O. Santolík ${ }^{2,1}$ \\ ${ }^{1}$ Institute of Atmospheric Physics, Bocni II/1401, 14131 Praha 4, Czech Republic \\ ${ }^{2}$ Charles University, Faculty of Mathematics and Physics, V Holesovickach 2, 18000 Praha 8, Czech Republic
}

Received: 23 June 2005 - Revised: 20 October 2005 - Accepted: 8 November 2005 - Published: 23 December 2005

\begin{abstract}
We investigate the ray trajectories of nonductedly propagating lower-band chorus waves with respect to their initial angle $\theta_{0}$, between the wave vector and ambient magnetic field. Although we consider a wide range of initial angles $\theta_{0}$, in order to be consistent with recent satellite observations, we pay special attention to the intervals of initial angles $\theta_{0}$, for which the waves propagate along the field lines in the source region, i.e. we mainly focus on waves generated with $\theta_{0}$ within an interval close to $0^{\circ}$ and on waves generated within an interval close to the Gendrin angle. We demonstrate that the ray trajectories of waves generated within an interval close to the Gendrin angle with a wave vector directed towards the lower L-shells (to the Earth) significantly diverge at the frequencies typical for the lower-band chorus. Some of these diverging trajectories reach the topside ionosphere having $\theta$ close to $0^{\circ}$; thus, a part of the energy may leak to the ground at higher altitudes where the field lines have a nearly vertical direction. The waves generated with different initial angles are reflected. A small variation of the initial wave normal angle thus very dramatically changes the behaviour of the resulting ray. Although our approach is rather theoretical, based on the ray tracing simulation, we show that the initial angle $\theta_{0}$ of the waves reaching the ionosphere (possibly ground) is surprisingly close - differs just by several degrees from the initial angles which fits the observation of magnetospherically reflected chorus revealed by CLUSTER satellites. We also mention observations of diverging trajectories on low altitude satellites.
\end{abstract}

Keywords. Magnetospheric physics (Waves in plasma) Space plasma physics (Wave-particle interactions, Waves and instabilities) - Ionosphere (Wave propagation)

\section{Introduction}

Together with the lightning induced whistlers, chorus emissions belong to the most distinct electromagnetic waves propagating in the inner magnetosphere. Chorus was first

Correspondence to: J. Chum

(jch@ufa.cas.cz) observed on the ground (Storey, 1953). Later its investigation has mainly been based on the satellite measurements. Chorus propagates as whistler mode plasma waves and consists of narrowband tones usually rising (sometimes falling) in frequency on the time scale of several tenths of a second. Near the magnetic equator, chorus occurs usually in two distinct frequency bands separated by a narrow gap at one half of the electron cyclotron frequency $\omega_{c e q}$ (Tsurutani and Smith, 1974), with the upper-band just above the half of $\omega_{\text {ceq }}\left(\omega / \omega_{\text {ceq }} \sim 0.5-0.6\right)$, and the lower band in the range $\left(\omega / \omega_{\text {ceq }} \sim 0.2-0.45\right)$.

The satellite measurements have recently brought new pieces of knowledge about chorus properties. The Poynting flux measurement on Polar (LeDocq et al., 1998) and the CLUSTER satellites (Parrot et al., 2003) have confirmed the previous suggestion by Helliwel $(1967,1969)$, that the chorus source is located close to the magnetic equatorial plane, with the dimension along magnetic field line being up to several thousands of kilometres, thus expanding a few degrees from the magnetic equator (Santolík et al., 2005). The crosscorrelation of spectrograms recorded on different CLUSTER satellites have shown that the transverse dimension (with respect to magnetic field) of the source located at the magnetic equator could be lower than $100 \mathrm{~km}$ (Santolík et al., 2004) in the case of lower band chorus. Beyond that distance the correlation coefficient calculated from the spectrograms rapidly decreases.

The detailed understanding of the generation mechanism is still a subject of active research. It is generally believed that the generation of chorus emissions is driven by the injection of substorm electrons (Tsurutani and Smith, 1974; Bespalov and Trakhengerts, 1986) that interact with whistler mode waves through the cyclotron resonance (Andronov and Trakhtengerts, 1964; Kennel and Petschek, 1966). However, the linear theory cannot clarify the discrete character of the chorus elements. Several authors have attempted to explain the main properties of the chorus emission, such as the recurrence rate and the slope of the chorus elements (tones). Trakhtengerts $(1995,1999)$ used a theory of the backward wave oscillator in the ELF/VLF frequency band. According to his theory, a step-like electron distribution function, which 
is formed as a result of the development of a cyclotron instability, leads to an absolute instability and the wave generation in the form of discrete elements with rising frequency. Nunn et al. (1997) considered that a strong nonlinear phase trapping of cyclotron resonant electrons is the essential mechanism producing the VLF chorus. Using the Vlasov code simulation in 4-D phase space, Nunn (2004) was able to reproduce the main properties of chorus.

Originally, the chorus properties have been studied under the assumption of ducted propagation of the emission from the source. This assumption has been supported by the fact that chorus emissions can be observed on the ground. Next, it has generally been accepted that the lower band chorus is generated with wave vectors directed close to the magnetic field lines. The latter assumption is also based on the satellite measurements carried out close to the source region, which show that the mean value of wave normal angle $\theta_{0}$ is usually small, close to zero (Nagano et al., 1996). However, the first identification of magnetospherically reflected (MR) chorus described by Parrot et al. (2003), and the plasma density measurements show that, in many cases, there are no ducts and chorus emissions may propagate obliquely with respect to magnetic field lines. The detailed ray tracing analysis of the MR chorus shows that the emission has to be generated with relatively large wave normal angles $\theta_{0}$ to the magnetic field line (Parrot et al., 2004), with the wave vector directed towards the Earth. Chum et al. (2003) showed, under the assumption of nonducted propagation, that a span of wave normal angles should exist in the generation region, so that it would be possible to observe a chorus element at higher latitudes.

Applying the ray tracing analysis to the frequencies that are typically observed both on the satellites and on the ground and assuming nonducted propagation, we will show that for specific values of initial angle $\theta_{0}$, a relatively small change in the initial angle $\theta_{0}$ leads to the distinctly different wave trajectories and to the divergence of wave normal angles along these trajectories. We will demonstrate that the divergence occurs for waves generated at relatively large angles, with wave vectors directed towards lower L-shells (the Earth). Some of these waves may reach the topside ionosphere with $\theta \sim 0$, thus probably leaking to the ground, whereas the waves generated with sufficiently different initial $\theta_{0}$, including those generated along the field line, undergo the magnetospheric reflection.

In the present paper we concentrate on a detailed theoretical ray tracing analysis of this problem. In a related experimental paper (Santolík et al., 2005¹ ), we present an interpretation of spacecraft observations of the divergent propagation pattern at high latitudes.

\footnotetext{
${ }^{1}$ Santolík, O., Chum J., Parrot M., Gurnett, D. A., Pickett, J. S., and Cornilleau-Wehrlin, N.: Propagation of whistler-mode chorus to low altitudes: Spacecraft observations of structured ELF hiss, J. Geophys. Res., submitted, doi:2005JA011462, 2005.
}

2 Convergence and divergence of ray trajectories; ground accessibility and magnetospheric reflection; results of ray tracing simulation

\subsection{Ray tracing}

In this section we will investigate the ray trajectories of lower band chorus waves for a wide range of initial angles $\theta_{0}$ between the wave vector and magnetic field line. The ray tracing software that we use is based on the dipole magnetic field model, which means that the cyclotron frequency $\omega_{c}$ is given by expression (1)

$\omega_{c}=\omega_{c 0} \cdot \frac{\left(1+3 \cdot \sin ^{2} \lambda\right)^{1 / 2}}{\left(R / R_{E}\right)^{3}}$,

where $\omega_{c 0}$ is the electron cyclotron frequency at the magnetic equator on the Earth's surface, $\lambda$ is magnetic latitude, $|\boldsymbol{R}|=L \cdot \cos ^{2}(\lambda)$, and $L$ is the distance of magnetic field line from the Earth's centre in the magnetic equator plane, in Earth radii $\left(R_{E}\right)$.

In order to verify the robustness of the acquired results, we have used two different packages of ray tracing software and compared the results. The first software uses a gyrotropic distribution of plasma density (plasma frequency $\omega_{p}$, respectively), that is $\omega_{p}^{2} \propto \omega_{c}^{n}(\mathrm{n} \sim 1)$, and an approximation of the general dispersion relation in cold magnetized plasmas, which is valid only in the frequency range $\omega_{c i} \ll \omega \leq \omega_{c} \cdot \cos \theta$ ( $\omega_{c i}$ is ion cyclotron frequency), and in a dense plasma, $\omega_{p}^{2} \gg \omega_{c}^{2}$. The latter inequality holds in most of the magnetosphere, except for the polar region. The effect of ions on whistler mode wave propagation is in the approximation of the dispersion relation (Eq. 2) included via the Lower Hybrid Resonance (LHR) frequency $\omega_{L H}$.

$\omega^{2}=\frac{\omega_{L H}^{2}}{1+\frac{\omega_{p}^{2}}{k^{2} \cdot c^{2}}}+\frac{\omega_{c}^{2} \cdot \cos ^{2} \theta}{\left(1+\frac{\omega_{p}^{2}}{k^{2} \cdot c^{2}}\right)^{2}}$,

where $c$ is speed of light and $k$ is the modulus of the wave vector. An analytic model of LHR frequency, which reproduces the main features of the LHR frequency in the plasmasphere is used. More details can be found in Shklyar and Jiricek (2000).

The second ray tracing software uses a diffusive equilibrium model of plasma density distribution, defined by the balance of the thermal pressure and gravitational forces. A temperature of $1000 \mathrm{~K}$ at the reference level of $1000 \mathrm{~km}$ has been used in this paper as a basis for the density model, together with predefined ratios of the plasma frequency to the electron cyclotron frequency in the chorus source region. Another difference compared to the previous raytracing software is that we do not use approximative expressions for the dispersion relation, but an exact solution to the dispersion relation in cold plasmas, taking into account both electron and ion contributions (Stix, 1992). Several ion species can be taken into account but only a hydrogen plasma is used in the present paper. The rays are generally followed in three dimensions, but this capability 

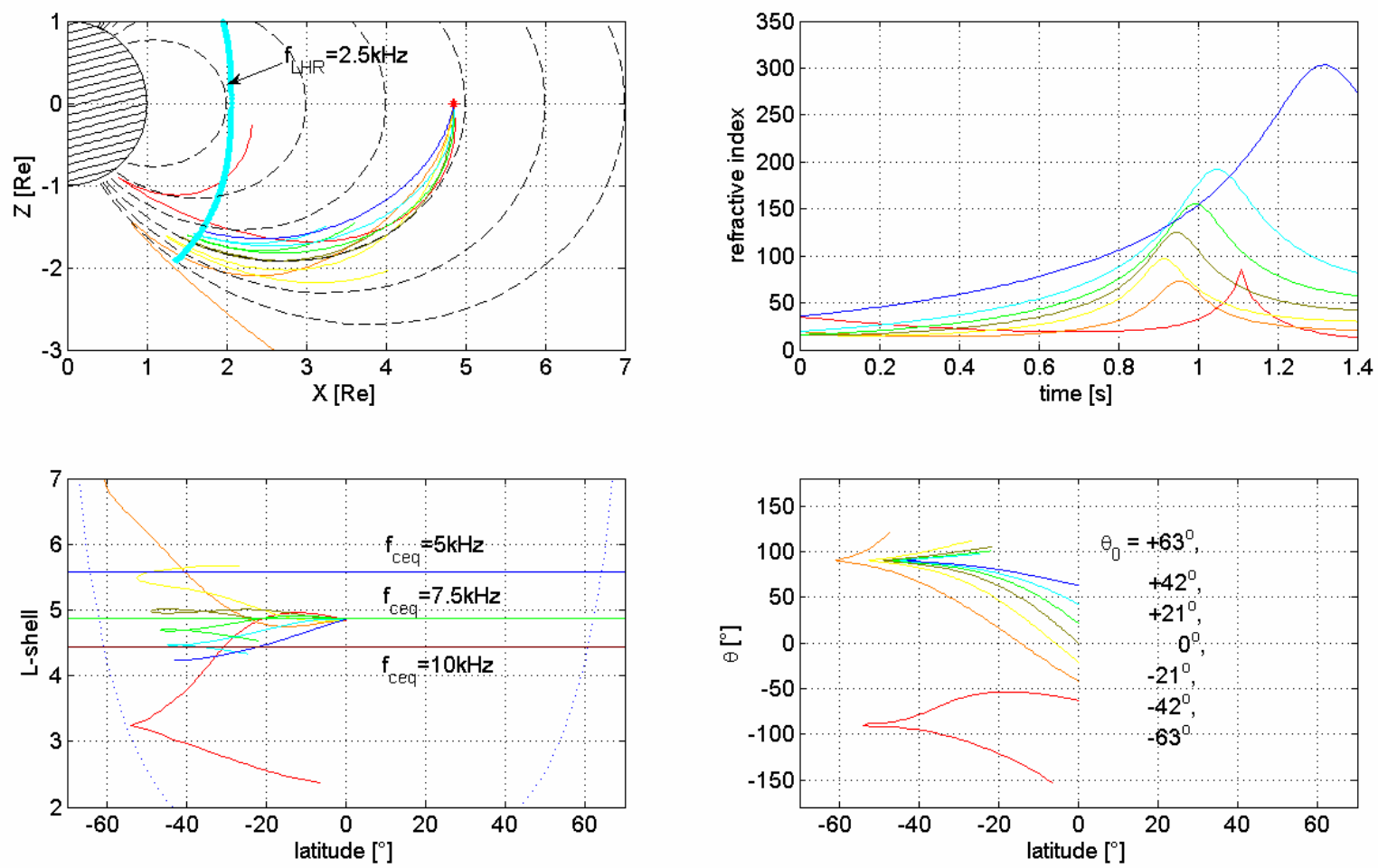

Fig. 1. Ray tracing analysis of $2.5 \mathrm{kHz}$ waves for 7 different initial angles generated at $L=4.85$. The gyrotropic model of plasma density distribution was used. Colours discern the different initial angles $\theta_{0}$; the values of the initial angles are written in the right bottom plot. Left column: ray trajectories in different coordinate systems; upper plot: in the meridian plane (xz plane, the $\mathrm{z}$ axis corresponds to the axis of the Earth's magnetic dipole), underneath: in magnetic latitude and L-shell coordinates. Right column: evolution of the refractive index as a function of time (on top), wave normal angle $\theta$ as a function of magnetic latitude (on the bottom). Here and further, the notation is the following: $\theta=0$ means that the wave propagates along the field line towards the Southern Hemisphere, the " + " sign stands for waves having the wave vector directed towards the higher L-shells, $\theta=180^{\circ}$ represents the wave propagating along the field line towards the Northern Hemisphere. In the top left plot, the thick cyan colour indicates the contour of $\omega_{L H}=2.5 \mathrm{kHz}$. In the bottom left plot, the blue, green and brown lines represent the equatorial electron cyclotron frequency of $5 \mathrm{kHz}, 7.5 \mathrm{kHz}$ and $10 \mathrm{kHz}$.

is not used here, and only two-dimensional calculations in the local magnetic meridian plane are performed. The basic properties of this software were presented by Cairo and Lefeuvre (1986). Compared to this described version, the algorithm of the integration of differential equations of the ray trajectories has been rewritten, using the Runge-Kutta 4th order method with one midpoint and an adaptive integration step. Additionally, wave-mode control and integration stop criteria have been modified. The Wentzel-Kramers-Brillouin (WKB) approximation of geometric optics is verified in every integration step and the ray is not followed beyond the limit where this basic approximation becomes invalid.

\subsection{Magnetospheric reflection}

Before we start the ray tracing simulation, it is instructive to keep in mind the basic characteristics of a magnetospheric reflection (MR), which is actually, strictly speaking, a refraction. Although it is related to the LHR frequency, it doesn't occur exactly at the point where the wave frequency equals the local LHR frequency $\omega=\omega_{L H}$, as it is often stated for simplicity, but may also happen at frequencies well below this value. The detailed analysis has recently been made by Shklyar et al. (2004), who studied the MR lightning induced whistlers observed close to the reflection region. Shklyar et al. (2004) showed that the reversal of the direction of the group velocity is mainly due to the reversal of its parallel component (with respect to the magnetic field), which takes place at the frequency $\omega$, given by Eq. (3), which may be obtained from the definition of group velocity and Eq. (2):

$$
\omega^{2}=\frac{\omega_{L H}^{2}}{1+\frac{\omega_{p}^{2}}{k^{2} \cdot c^{2}}} .
$$

From Eq. (3), it is obvious that the frequency $\omega$, at which the parallel component of the group velocity reverses, is always less than the local LHR frequency $\left(\omega<\omega_{L H}\right)$, and approaches the LHR frequency as the wave number increases, being approximately equal to it for waves propagating close to the resonance cone.

\subsection{Initial conditions and assumptions}

The initial conditions and assumptions in our analysis are the following: 

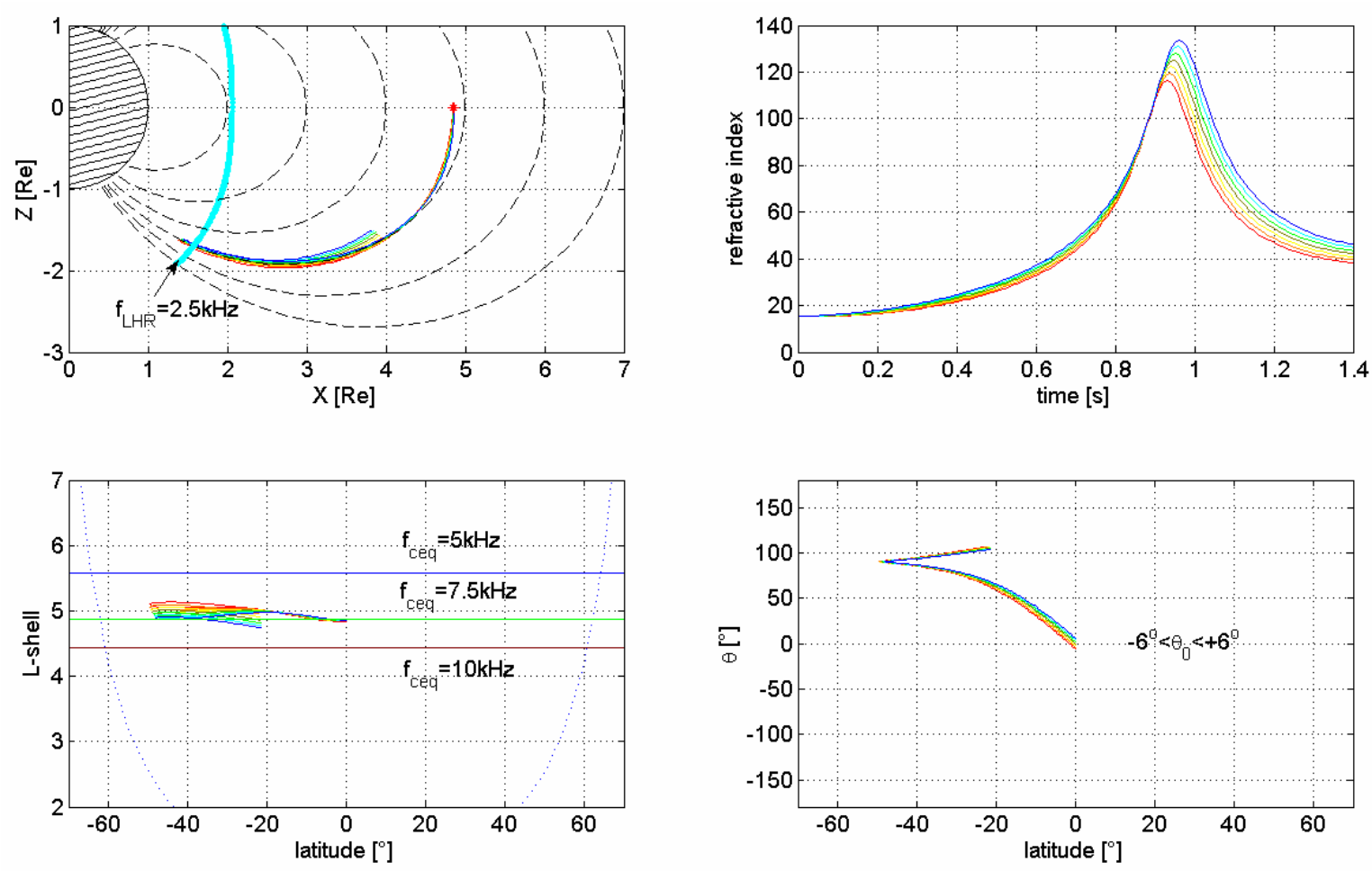

Fig. 2. Ray tracing analysis of $2.5 \mathrm{kHz}$ waves generated at $L=4.85$ having the initial angles along the field line in the range $-6^{\circ} \leq \theta \leq+6^{\circ}$, that is, with the wave vector initially directed along the field line. The gyrotropic model of plasma density distribution was used. We can see that these rays undergo magnetospheric reflection and don't diverge significantly. The rays also don't change the initial L-shell distinctly. The colours change with increasing initial angle $\theta$, similarly as in Fig. 1 .

- Nonducted propagation; there are no sharp density gradients;

- A small transverse dimension of the source region (Santolík and Gurnett, 2003);

- There is a span of initial wave normal angles $\theta_{0}$ in the source region;

- We will show the analysis for two different frequencies: $2.5 \mathrm{kHz}$ and $575 \mathrm{~Hz}$. Initially, the gyrotropic model of plasma density distribution is applied in the analysis, however, the main subject of this paper, the divergence of trajectories, is presented for the two different models of plasma density distribution, in order to demonstrate the robustness of the result.

\subsection{Ray trajectories for a wide range of initial angles $\theta_{0}$}

We will start with waves of frequency $2.5 \mathrm{kHz}$ that are commonly observed both at ground stations and on satellites. Burtis and Helliwell (1976) have found in their statistical survey based on OGO 3 data that the normalized frequency distribution of mean chorus frequency has a peak slightly above $f / f_{c}=1 / 3$ in the equatorial region $\left(f_{c}\right.$ is the electron cyclotron frequency). Therefore, we will start our simulation in such a way that the initial ratio of $f / f_{c}$ at the equator is close to that peak, $\left(f / f_{c}=0.328\right.$ in our case $)$, at $L=4.85$. The important angles at the equator are the following: the resonance angle $\theta_{R}=70.85^{\circ}$, the Gendrin angle $\theta_{G}=49^{\circ}$.

First we study the ray trajectories for a wide interval of initial angles $\theta_{0}$, from $-63^{\circ}$ to $+63^{\circ}$ in the meridian plane. The " + " sign means that the wave vector is directed to higher L-shells (from the Earth). The simulation was first done for seven $\theta_{0}$ values with a relatively large step of $21^{\circ}$, so that we could easily distinguish the trajectories. The results are presented in Fig. 1; different colours are used to discern the trajectories corresponding to the different initial angles $\theta_{0}$. We can see that for most initial angles, the waves undergo magnetospheric reflection close to the region, where their frequency matches the LHR frequency. Note that even the waves which have been initially started along the field line $\left(\theta_{0}=0\right)$ do reflect magnetospherically. It can, however, be difficult to observe these reflected waves, because their trajectories merge with the trajectories of the more intense direct waves. Although there may be a transitional behaviour due to the existence of the Gendrin angle, we can see that the final L-shell usually decreases with an increasing initial angle $\theta_{0}$ (including the sign). There is, however, an exception for a large negative initial angle $\theta_{0}$. The trajectories started with initial angles $\theta_{0}$ between $-63^{\circ}$, and $-42^{\circ}$ are highly diverging (not shown). It should also be noted that the divergence is well expressed in the evolution of wave normal 

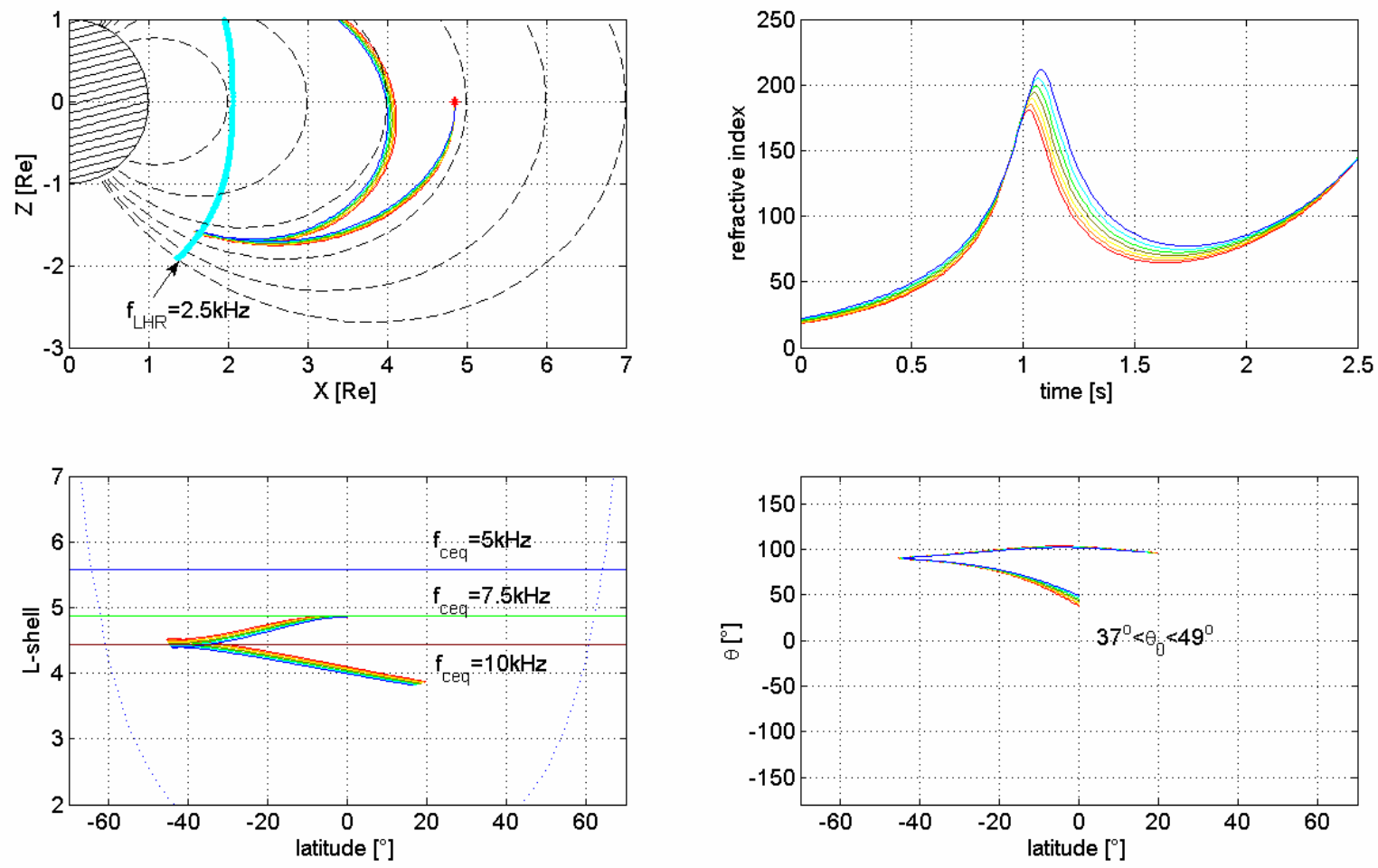

Fig. 3. Ray tracing analysis of $2.5 \mathrm{kHz}$ waves generated at $L=4.85$ having the initial angles in the interval $37^{\circ} \leq \theta \leq 49^{\circ}\left(\theta_{G}-12^{\circ} \leq \theta \leq \theta_{G}\right)$, that is, with the wave vector initially directed from the Earth, towards the higher L-shells. The gyrotropic model of plasma density distribution was used. We can see that the rays converge. The rays undergo the magnetospheric reflection, as well. Also remarkable is the decrease in the L-shell during propagation. At higher latitudes, the frequency normalized to the equatorial electron cyclotron frequency is about $1 / 4$. In the equatorial region, the rays propagate along the field line. The usage of colour is the same as in the previous figures.

angles $\theta$ along the trajectories, as well (right bottom plot). Another important feature is that these diverging trajectories arrive close to the topside ionosphere. The divergence of trajectories will be studied in more detail in the next subsection.

Note also that except the interval of initial angles corresponding to the diverging trajectories, the higher (positive) angle $\theta_{0}$ that we choose, the lower the L-shell of the ray trajectory at higher altitudes or after the magnetospheric reflection.

2.5 Ray trajectories of waves generated with group velocity along the field line

Santolík and Gurnet (2003), in their correlation analysis of chorus wave packets recorded on different CLUSTER satellites in the generation region, assumed that the emissions propagate along the field line in the equatorial region. This is also consistent with the theoretical assumption that there should be a sufficient length of interaction region, in which the waves propagate antiparallel to energetic electrons, so that the feedback between waves and particles could operate to produce discrete emissions. Usually a generation with wave vector along the field line is considered (e.g. Trakhtengerts, 1995, 1999; Nunn, 2004). However, the generation with a wave vector deviated from the field line to a value, which is close to the Gendrin angle (Gendrin, 1961) also results in the wave propagating along the field line in the generation region. More precisely, to ensure the initial propagation along the field line, the waves should be generated with angles a little bit smaller than the Gendrin angle, since the wave normal angle $\theta$ always tends to increase near the equatorial region during the nonducted propagation (see, for example, Figs. 1-5). In the following, we will analyse all three possible situations in the meridian plane: generation along the field line, generation close to the Gendrin angle with a wave vector directed to higher L-shells (from the Earth) and with the wave vector directed to the lower L-shells (toward the Earth). The results of the ray tracing simulations for waves of frequency $2.5 \mathrm{kHz}$ are presented in Figs. 2, 3, 4 (for the gyrotropic model of the plasma density distribution) and in Fig. 5 (for the diffusive equilibrium model of the plasma density distribution). Comparing the results of these simulations, we can see that the waves which started with positive angles $\theta$ (Fig. 3) exhibit the highest refractive index from these three possibilities of different intervals of initial angles $\theta_{0}$. They also converge most significantly; their ray bundle width is the smallest. Their energy density is thus the highest, especially in the electric component. Since their refractive index is high, it is probable that these waves would be damped significantly. They undergo the magnetospheric reflection in the region, where their frequency is very close 

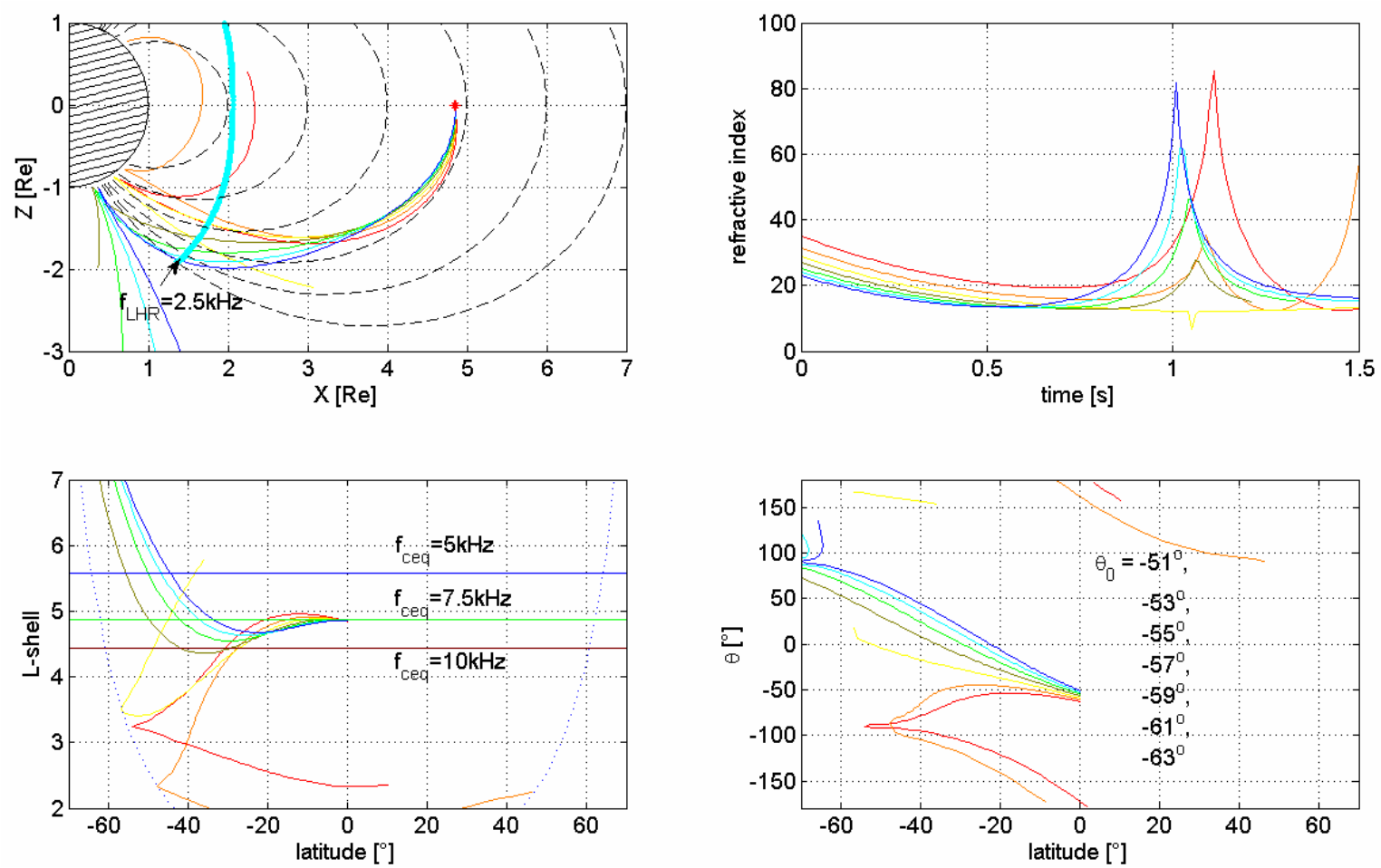

Fig. 4. Ray tracing analysis of $2.5 \mathrm{kHz}$ waves generated at $L=4.85$ having the initial angles in the interval $-63^{\circ} \leq \theta \leq-51^{\circ}$. The gyrotropic model of plasma density distribution was used. The divergence of ray trajectories and the divergence of the evolution of wave normal angles along these trajectories can be clearly seen. Most of the waves reach the topside ionosphere; thus, a part of their energy may possibly leak to the ground. The waves reaching the height of $\sim 300 \mathrm{~km}$ are artificially reflected in the simulation. The proper treatment of trajectories of these waves would require the full wave solution. The usage of colour is the same as in the previous figures.

to the local LHR frequency. The L-shell decreases substantially during propagation, except for the generation region. We should stress that the waves generated along the field lines are also magnetospherically reflected. However, they don't change the L-shell significantly (Fig. 2).

Starting with the wave vector directed significantly towards the lower L-shells we obtain completely different behaviour of the trajectories. The result of the simulation is in Fig. 4 (gyrotropic model of the distribution of plasma density) and in Fig. 5 (diffusive equilibrium model of plasma density distribution). First of all, we notice the remarkable divergence of the trajectories. Additionally, some of the waves generated with negative angles $\theta$ reach the topside ionosphere with relatively low angles $\theta$. Note that the ray trajectories are shown only for selected initial angles. However, all the trajectories between the displayed ones in the selected interval are considered in the discussions throughout the paper.

If the waves propagate approximately perpendicularly to the bottom boundary of the ionosphere, they can fulfill the condition for refraction/mode transfer to the "free space" electromagnetic waves, and reach the ground. If we assume, for simplicity, that the boundary between the bottom side of the ionosphere and the non-ionised atmosphere is a flat plane, then the boundary condition requires the tangential components of electric and magnetic fields to be continuous at this boundary. Under another assumption that the regions on both sides of the boundary can be considered as homogenous (the wave vector changes significantly on scales much larger than the wavelength), we can simply apply Snell's law, which states the equality for the tangential components of the refractive index $N$. Since the value of the refractive index in the non-ionised atmosphere is $\sim 1$, the value of the tangential component of the refractive index (with respect to the boundary) of the incident wave should also be close to $\sim 1$. Assuming that the boundary is parallel to the Earth's surface, and using the dispersion relation and proper plasma density, it is possible to estimate the maximum deviation of the wave vector from the vertical direction in the ionosphere, which can have the waves reaching the ground. (See also Fig. 6, presenting the components of refractive index in a magnetised cold plasma.) The waves propagating at higher angles to the vertical direction undergo the reflection back to the ionosphere. Thus, a small wave-normal angle with respect to the vertical direction prevents the ionospheric reflection, while a small angle to the magnetic field line prevents the magnetospheric reflection. It is obvious that both requirements can be fulfilled at higher magnetic latitudes, where the field and vertical direction are close to each other. In reality, the rapid change in the wave vector in the ionosphere might break the limitation of geometrical optics, and the exact treatments of such waves would require the full wave 

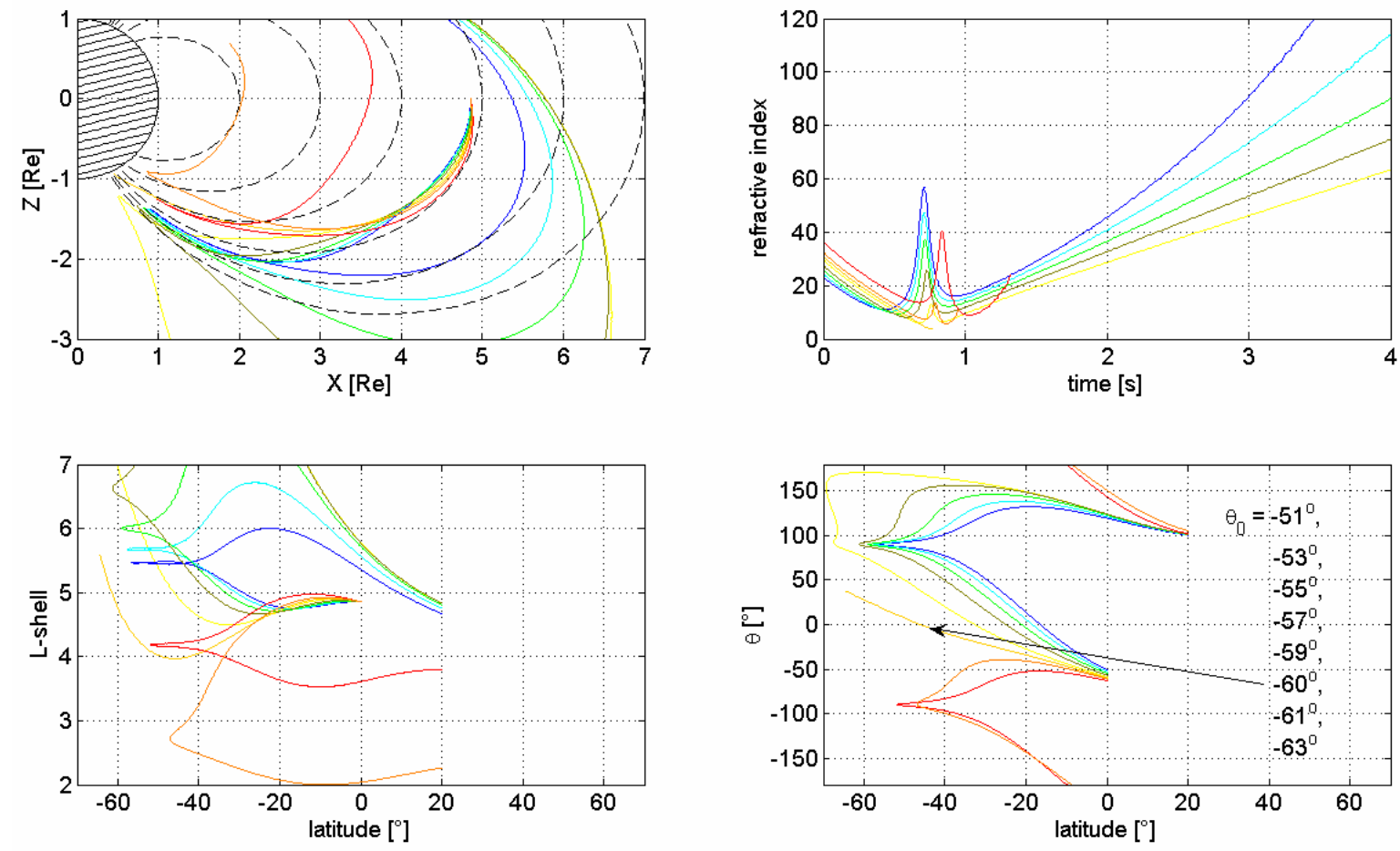

Fig. 5. The same as in Fig. 4, but for the diffusive equilibrium model of plasma density distribution.

solution. In the simulation, the waves reaching the height of $300 \mathrm{~km}$ are artificially reflected in Fig. 4. For the waves of frequencies below the proton cyclotron frequency the situation is even more complicated, and the multi-ion cutoff (Gurnett and Burns, 1968) should be considered for waves which do not propagate along the field line. The scattering on the irregularities in the ionosphere is also possible.

Let us call the bifurcation angle $\theta_{\mathrm{B}}$ the initial angle $\theta_{0}$, for which the trajectories arrive at the topside ionosphere with $\theta=0$. In the case presented in Fig. 4 , the bifurcation angle is a little bit less than $-59^{\circ}$. If we use the diffusive equilibrium model (Fig. 5), the bifurcation angle is a little bit less than $-60^{\circ}$. From the simulation, it is obvious that if the initial angle approaches the bifurcation angle the trajectories tend to diverge most significantly. For the initial angle $\theta_{0}>\theta_{\mathrm{B}}$ the trajectories tend to reflect towards higher L-shells, whereas for the $\theta_{0}<\theta_{\mathrm{B}}$ the trajectories tend to reflect towards lower L-shells. Of course, the bifurcation angle depends on the plasma density distribution, and the starting point from which we investigate the trajectories.

The existence of the bifurcation angle (divergence) of the trajectories is a consequence of the rather complicated dependence of the direction of the group velocity on the angle $\theta$, and the evolution of the wave vector along the ray trajectories for these initial conditions. For the clearness of further discussion, Fig. 6 displays the contours of $\omega=$ const in the $\left(N_{\perp}, N_{\|}\right)$space, or, in other words, the dependence of the refractive index (wave vector) on the angle $\theta$. The ray direction (direction of group velocity) can be found as the normal to this curve in a given point. The remarkable divergence and/or "temporally" convergence of the trajectories can be observed if the ray bundle consists of waves having angles around $\theta=0$ and/or around $\theta=\theta_{G}$; the latter possibility only exists in the frequency range $\omega_{L H}<\omega<\omega_{C} / 2$. The first case $(\theta=0)$ usually leads to the divergence of the trajectories (provided that $\left.\omega<\omega_{c} / 2\right)$ in the Earth's magnetosphere, whereas the occurrence of the second case $\left(\theta \sim \theta_{G}\right)$ is often connected with the intersection of the trajectories. Returning to our simulation results, we can see this intersection at latitudes from $\sim-20^{\circ}$ to $-25^{\circ}$ in Figs. 4 and 5. At higher latitudes and lower altitudes the waves reach the region where their frequency is $\omega<\omega_{L H}$, and they tend to reflect magnetospherically if their wave-normal angle is sufficiently high (Sect. 2.2). Some waves from the ray bundle propagate to the region where their frequency is considerably lower than $\omega_{L H}$ and the evolution of their wave normal angle inherently tends to 0 . All the other waves have a distinct tendency to reflect/to shift their wave vectors into the perpendicular direction to magnetic field lines. Simultaneously, we can observe an extremely remarkable divergent propagation; the evolution of wave-normal angles is highly divergent, as well. This is what we call divergence further on in this paper, though the trajectories are diverging, in fact, from the beginning. The most distinct divergence occurs for waves whose initial angles approach the value of the bifurcation angle. Note that for most of the initial angles there is also an overall tendency of the oblique whistler mode wave to steer its wave vector towards higher L-shells as it propagates from the equator in the inner magnetosphere (see, for example, Fig. 1). 

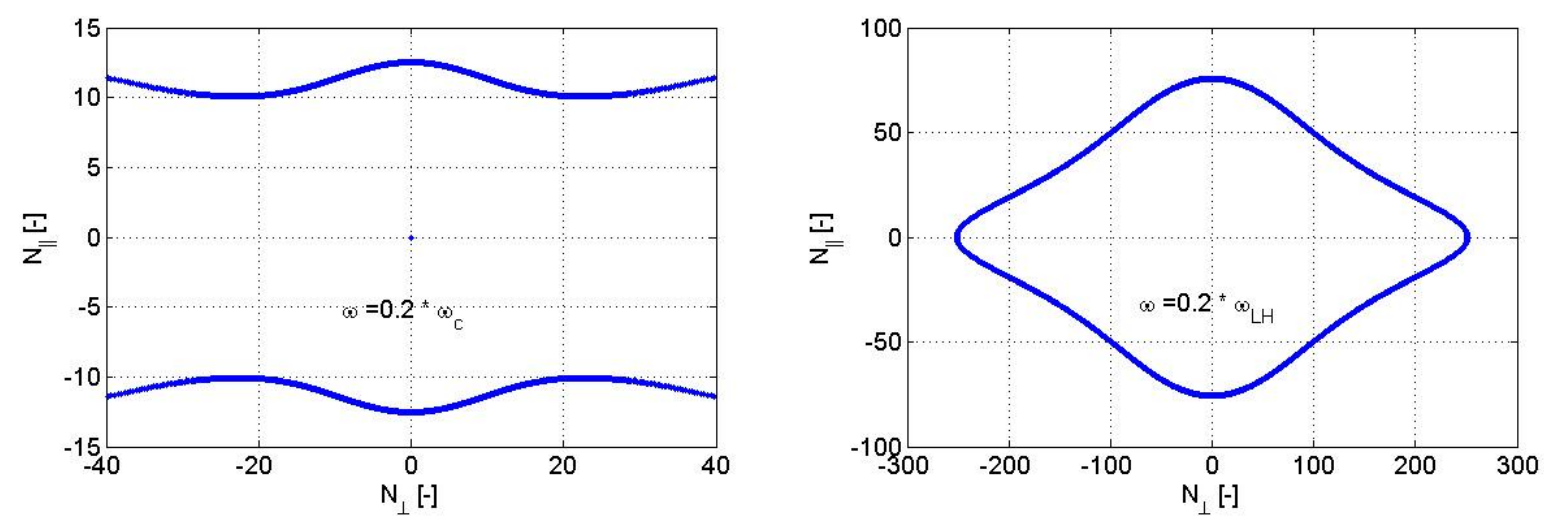

Fig. 6. Contours of $\omega=$ const in the space of perpendicular $\left(N_{\perp}\right)$ and parallel $\left(N_{\|}\right)$components of the refractive index with respect to the magnetic field line. The direction of the group velocity is given by the normal to these contours. Left plot displays the situation for the frequency range $\omega_{L H}<\omega<\omega_{c} / 2$; the right plot shows the case for frequencies $\omega_{c i}<\omega<\omega_{L H}$. Note that the scale is different in both plots and axis. Both plots were computed under the assumption $\omega_{p} / \omega_{c}=5$.
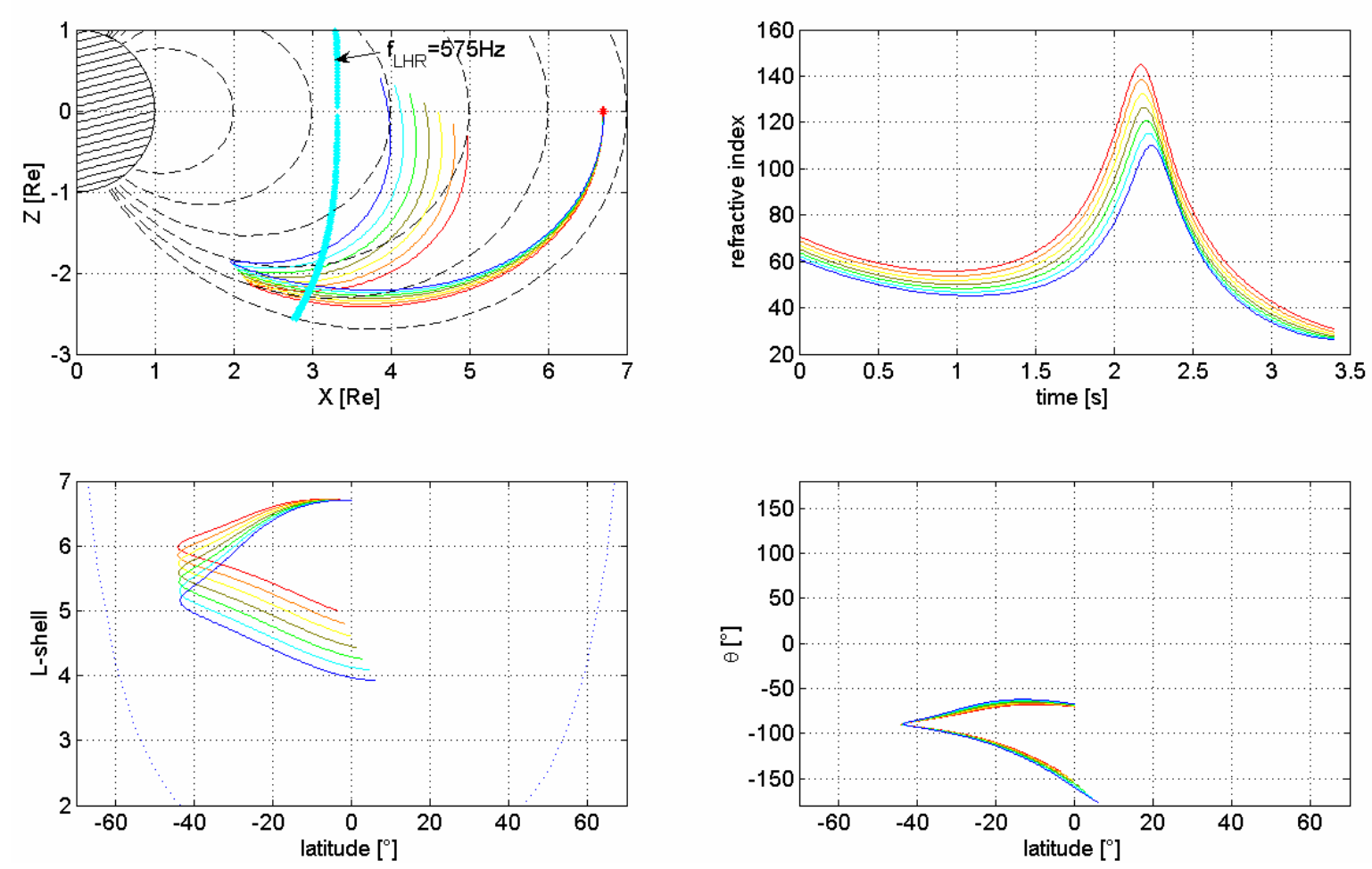

Fig. 7. Ray tracing analysis of MR chorus observed by Parrot et al. (2003). The gyrotropic model of plasma density distribution was used. The analysis was started at the equator at $L=6.7$. The initial angles are in the interval $-70.5^{\circ} \leq \theta \leq-67.5^{\circ}$. The usage of colour is the same as in the previous figures. This means, for example, red line stands for $\theta_{0}=-70.5^{\circ}$, the blue line stands for $-67.5^{\circ}$, the olive line stands for $-69^{\circ}$.

\subsection{Observed MR chorus and divergence of ray trajectories}

The bifurcation angle, introduced in the previous paragraph, is close to the Gendrin angle and corresponds to wave vectors directed towards the lower L-shells (to the Earth). Although the main portion of chorus energy could be generated in different wave vector directions, it is surprising that similar initial conditions give the ray trajectories that correspond to the observation of MR chorus described by Parrot et al. (2003). Therefore, we will continue with the ray tracing analysis of MR chorus observed simultaneously with direct chorus (propagating from the equator) by Parrot et al. (2003). Let us shortly summarize the facts: the direct chorus waves were observed on the CLUSTER satellites at a radial distance 

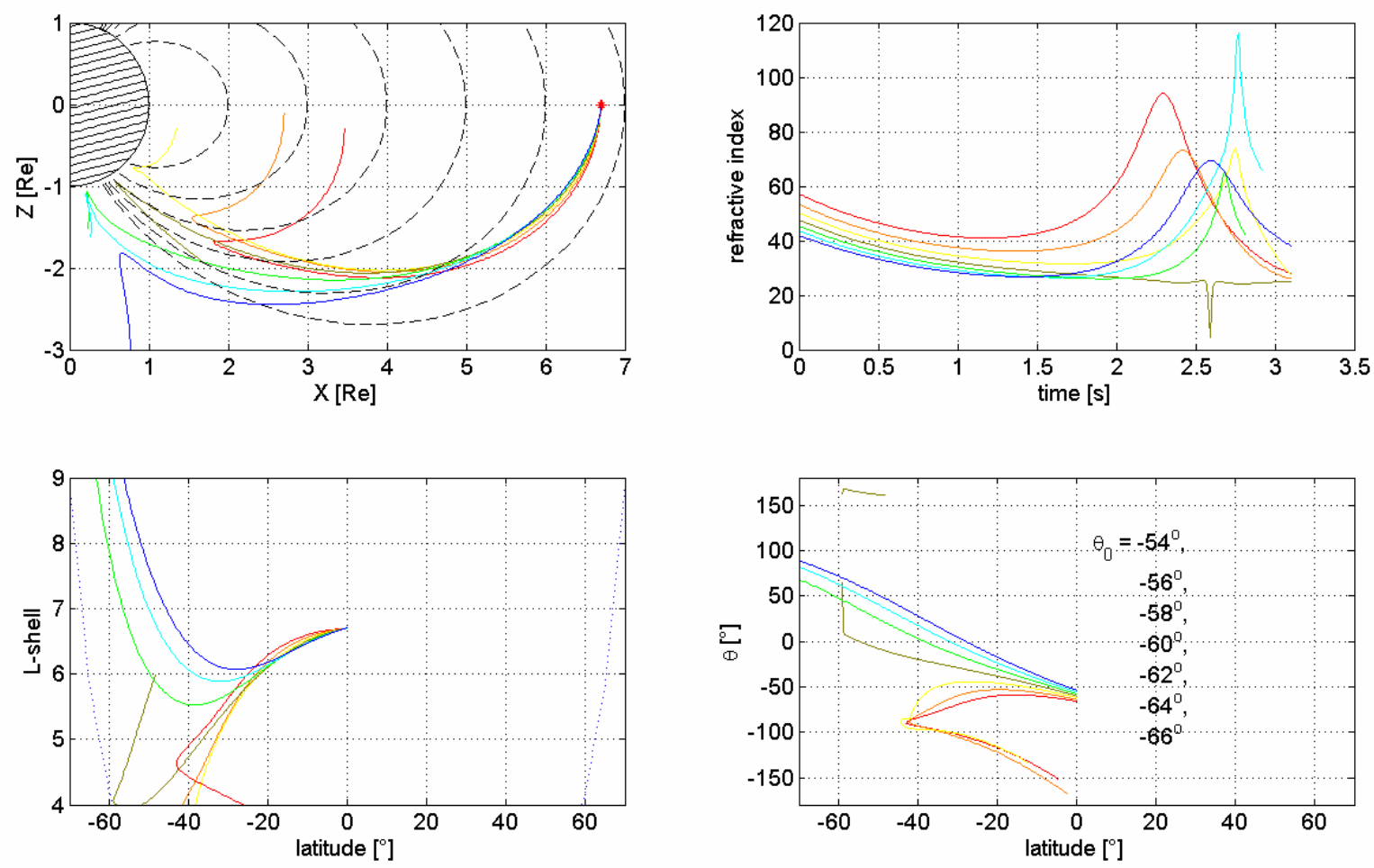

Fig. 8. Ray tracing analysis of $575 \mathrm{~Hz}$ waves starting at the same place as in Fig. 7, but having the range of initial angles $-66^{\circ} \leq \theta \leq-54^{\circ}$. The gyrotropic model of plasma density distribution was used. The divergence of ray trajectories and the divergence of wave normal angles along the ray trajectories can be clearly seen. The colours again indicate the different initial angles $\theta_{0}$. Assignment of colours is obvious from the right-hand panel at the bottom; the system is the same as in the previous figures. The waves reaching the height of $300 \mathrm{~km}$ are artificially reflected in the simulation. The proper treatment of trajectories of waves reaching the topside ionosphere would require a full wave approach.

of $4.5 R_{E}$ ( $R_{E}$ is the Earth's radius) and magnetic latitude MLAT $\sim 35^{\circ}(\mathrm{L} \sim 6.7)$, simultaneously with MR chorus $(2-3$ orders weaker) at $\mathrm{R} \sim 4.4$ and MLAT $\sim 10^{\circ}(\mathrm{L} \sim 4.5)$. All satellites were located close to one single meridian plane. The result of the simulation (in the meridian plane) that more or less fits this observation is graphically shown in Fig. 7. The waves of frequency $575 \mathrm{~Hz}$ were launched at the magnetic equator at $L=6.7$ within the range of initial wave normal angles $-70.5^{\circ} \leq \theta_{0} \leq-67.5^{\circ}$. The model electron cyclotron frequency at the equator is $2.89 \mathrm{kHz}$; thus, the initial ratio $\mathrm{f} / \mathrm{fc}=0.1989$ at the equator is distinctly lower than in the previous case. The resonance angle $\theta_{R} \sim \operatorname{acos}(\mathrm{f} / \mathrm{fc})$, and the Gendrin angle $\theta_{G} \sim \operatorname{acos}(2 \mathrm{f} / \mathrm{fc})$ at the equator are the following: $\theta_{R}=78.53^{\circ}, \theta_{G}=66.56^{\circ}$. We can see that after the initial phase, when the rays propagate approximately along the field line, the rays undergo a rapid decrease in the L-shell (the bottom panel on the left) and return to the equator propagating almost anti-parallel to the magnetic field lines.

Worth noticing is also the fact that the trajectories are quite sensitive to the initial angle $\theta_{0}$, although the divergence of trajectories is not so distinct as it was in the case presented in Figs. 4 and 5. Now there is nearly no divergence of angle $\theta$ along the trajectories. Nevertheless, based on the experience with the previous analysis for waves of frequency $2.5 \mathrm{kHz}$, it is reasonable to suppose that the highly divergent pattern may occur for initial angles $\theta_{0}$ which are not too far from the interval $-70.5^{\circ} \leq \theta_{0} \leq-67.5^{\circ}$. Indeed, the simulations presented in Fig. 8 (gyrotropic model of plasma distribution) and in Fig. 9 (diffusive equilibrium model of plasma distribution) show that also in these cases we can find a highly divergent pattern both in the trajectories and in the wave normal angle $\theta$. The bifurcation angle is approximately $-60^{\circ}$ for the gyrotropic model, or somewhat more than $-62^{\circ}$ in the case of the diffusive equilibrium model of the plasma density distribution. In both cases, these angles are not too far from the initial angle corresponding to chorus rays shown in Fig. 7, which fit rather well the observations of MR chorus described by Parrot et al. (2003), and at the same time, from the value of the Gendrin angle $\left(\theta_{G}=66.56^{\circ}\right)$.

Note that, for initial angles $\theta_{0}$, which are more deviated from the field line towards lower L-shells than the bifurcation angle $\theta_{B}$, the magnetospheric reflection gradually starts to take place. The larger the deviation is, the higher refractive index and the closer the waves reflect to the region where $\omega=\omega_{L H}$. The waves whose initial deviations (angles) are only somewhat larger than $\theta_{B}$ reach relatively low L-shells (see, for example, the orange line in Fig. 8). Note that the simulation was done under the assumption of the absence of sharp density gradients. If the chorus source is located well outside the plasmapause, and the plasmapause is only weakly 

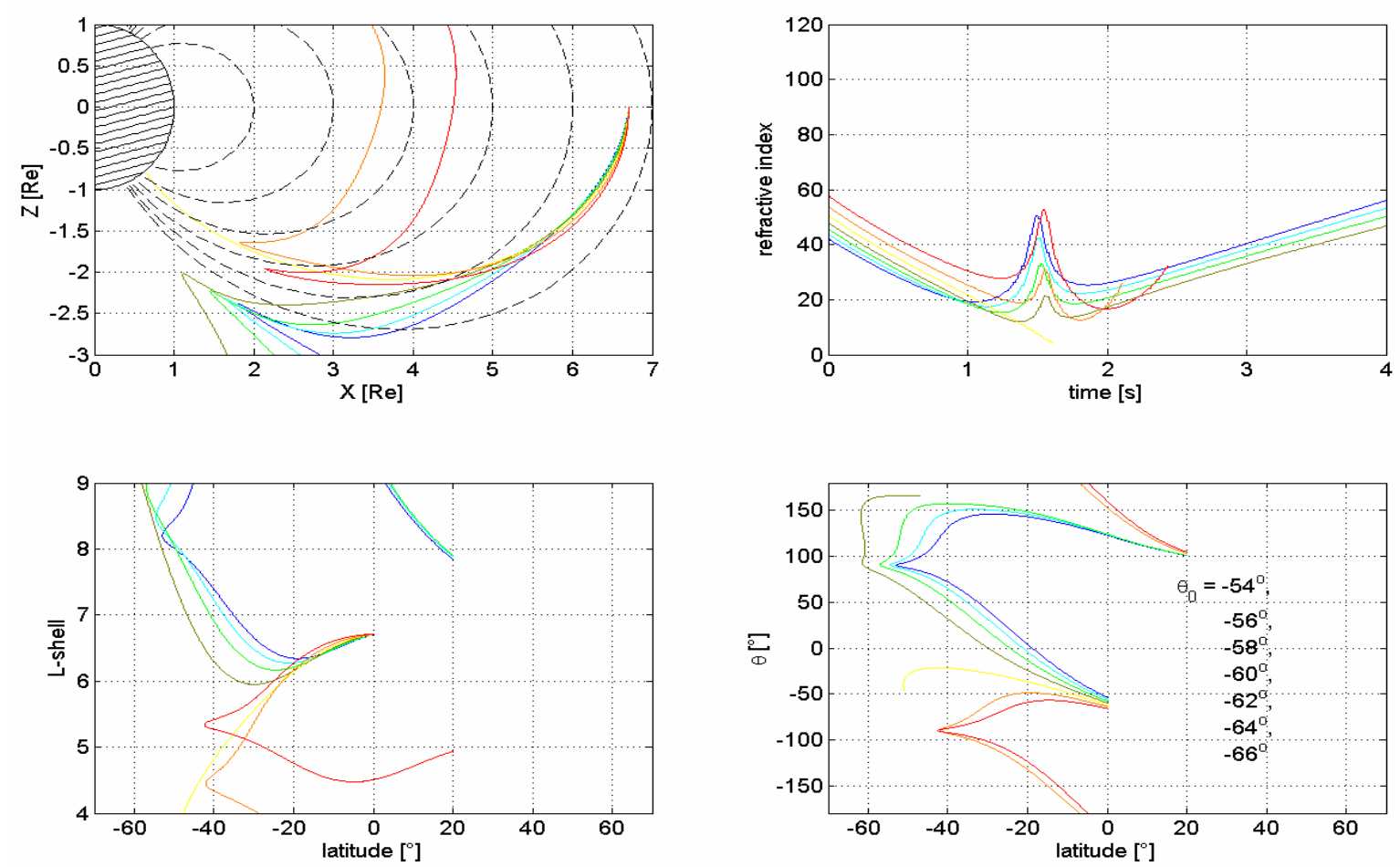

Fig. 9. The same as in Fig. 8, but for the diffusive equilibrium model of the plasma density distribution.

expressed at higher latitudes (if it is present at all), we can speculate that these waves can propagate into the plasmasphere. The analysis of wave propagation in the presence of distinct density gradients is out of scope of the present article.

\section{Discussion, observations and general remarks}

If we compare the results obtained by using different plasma density models, we can see that the trajectories differ, but the resulting pattern/behaviour is about the same. Since we obtain similar results for different frequencies, for a different initial ratio $f / f_{c}$, and for a different plasma density distribution, the diverging trajectories should be observed in nature, provided that the chorus waves are generated with the appropriate initial angles. The observations of MR chorus (Parrot et al., 2003, 2004) are probably one of the experimental examples of chorus which might be generated with sufficiently large initial angles.

Other observations, which are consistent with the existence of diverging trajectories, are the measurements of ELF waves by Santolík and Parrot $(1999,2000)$ on the lowaltitude satellite FREJA. As the satellite moved through the subauroral region from lower magnetic latitudes (MLAT), it observed that intense downgoing waves first had wave vectors inclined toward the equator. Then the spacecraft moved to higher latitudes, and at MLAT $~ 62^{\circ}$, the downgoing waves started to be observed with wave vectors in- clined poleward. This is qualitatively in agreement with the divergent propagation pattern shown by our ray tracing results presented in Figs. 4, 5, 8, and 9.

In a related experimental paper (Santolík et al., 2005) ${ }^{1}$ we show that reverse ray tracing, based on the observed wave vectors of ELF hiss at low altitudes, indicates a possible source region near the geomagnetic equator at a radial distance between 5 and $7 R_{E}$, and a generation mechanism acting on highly oblique wave vectors near the local Gendrin angle. Analysis of waveforms received at altitudes of $700-1200 \mathrm{~km}$ by the Freja and DEMETER spacecraft shows that low-altitude ELF waves contain discrete time-frequency structures resembling wave packets of whistler-mode chorus. Detailed measurements of the CLUSTER spacecraft at radial distances of 4-5 $R_{E}$ show chorus propagating downward from the source region localized close to the equator. The time-frequency structure and frequencies of chorus observed by CLUSTER along the simulated reverse ray paths of ELF hiss are consistent with the hypothesis that the frequently observed dayside ELF waves are just the low-altitude manifestation of natural magnetospheric emissions of whistler-mode chorus.

Note that the waves/trajectories corresponding to the initial angles $\theta_{0}$ which are less than the bifurcation angle $\theta_{B}$ (in absolute values greater, with wave vectors directed more distinctly to lower L-shells) may, after reflection, fill the plasmasphere (see Figs. 1, 4, 5, 8, 9) and might be one of the possible sources of plasmaspheric hiss. We should 
remark here that the remnants of MR lightning induced whistlers form another - probably most important source of plasmaspheric hiss (Green et al., 2005; Storey et al., 1991; and references therein), not excluding the possibility that plasmaspheric hiss is directly generated or amplified at high altitudes in the plasmasphere by the cyclotron resonance with energetic electrons.

It seems improbable that most of the chorus energy is radiated with wave vectors deviated by the bifurcation angle or by angles close to the Gendrin angle, oriented towards the lower L-shells; nevertheless, the distribution of chorus energy with respect to wave vector orientation remains one of the unanswered problems. As we have mentioned earlier, from the fact that near the equator the emissions seem to propagate along the field line, we deduce that most of the energy should be generated either along the field lines with $\theta \sim 0$ or with $\theta \sim \theta_{G}$. This is also consistent with the theoretical assumption that there should be a sufficient length of interaction region between the waves and the energetic electrons, so that the source could generate emissions of a discrete character. Although most of the authors consider the generation along the field line to be in operation, we cannot exclude that a significant part of the energy of the observed chorus emissions on the satellite comes from the waves that were generated close to the Gendrin angle. The resonant energy for the commonly considered fundamental cyclotron resonance is, however, higher than in the case $\theta \sim 0$, but not dramatically. It should be mentioned here that in the case of oblique waves, the Landau (Cerenkov) resonance may also play an important role, and that the Landau resonance energy is lower than the cyclotron one for the waves of frequencies $f / f_{c}<1 / 2$. Note that the Landau resonance does not exist for the waves having exactly $\theta=0$, because the wave is then purely transverse and has no component of the electric field along the wave vector. For highly oblique angles, approaching the resonance cone, the higher order cyclotron interactions can also contribute to the over-all growth rate. Whether the wave is damped or amplified depends on the electron distribution function and on the net results of all resonances. The stability of obliquely propagating whistlers was discussed, for example, by Brinca (1972).

The reason for the suggestion that a considerable part of the energy is generated at higher angles $\theta$ is the evolution of $f / f_{\text {ceq }}$ in dependence on the magnetic latitude observed on the satellites (CLUSTER, MAGION 5, POLAR), which shows that the nonducted emissions move to lower L-shells as they propagate from the equator to the middle or higher latitudes. An example can be found in Chum et al. (2003). Note that Lauben et al. (2002) also came to the conclusion that a lower band chorus is generated with $\theta \sim \theta_{G}$ when fitting the ray tracing procedure on chorus observed by the POLAR/PWI receiver. The exact treatment of this problem, is however, worth further and more detailed study because the initial $f / f_{\text {ceq }}$ has never been known precisely. The difference between the dipole magnetic field model used in the ray tracing and the real magnetic field, and the difference between the model plasma density distribution and the actual density distribution, may also cause significant uncertainty in the results. The encompassment of the third dimension may also play a role.

\section{Summary}

The ray tracing analysis shows that the trajectories of obliquely propagating chorus waves may exhibit a divergent behaviour accompanied by the divergence/bifurcation of the evolution of the wave normal angle $\theta$ along these trajectories. The initial angles $\theta_{0}$ at the equator, for which the divergence occurs depends on the initial $f / f_{c}$ ratio, plasma density distribution and magnetic field. For an initial angle $\theta_{B}$, which we call the bifurcation angle, the wave reaches the topside ionosphere having $\theta=0$. The value of the bifurcation angle is usually not too far from the value of the Gendrin angle, provided that the wave vector is directed towards the lower L-shells. The waves generated very close to the bifurcation angle may arrive at the ground, especially at higher latitudes, where the field lines have a nearly vertical direction. If the initial wave vector is deviated from the magnetic field line towards lower L-shells by an angle larger than $\theta_{B}$, the wave may, after reflection, propagate into the plasmasphere and contribute to the plasmaspheric hiss. If the initial angle differs from $\theta_{B}$ even more, the magnetospheric reflection occurs at higher altitudes. This was most probably the case of MR chorus observed by Parrot et al. (2003).

It is worth noticing that the magnetospheric reflection also takes place for the waves generated along the field lines $(\theta \sim 0)$, providing nonducted propagation.

Acknowledgements. This study was supported by the grant 205/03/0953-2-03 of the grant agency of the Czech Republic and by the ESA PECS contract No. 98025. The authors would like to thank also E. Titova from the Polar Geophysical Institute, Apatity, Russia, for valuable discussions on the related topics, D. Shklyar from IZMIRAN, Russia for the ray tracing software, and M. Parrot from LPCE/CNRS, Orleans, France, for fruitful discussions on MR chorus observations and ray tracing. The authors are also grateful both referees for their improving comments.

Topical Editor T. Pulkkinen thanks D. Shklyar and another referee for their help in evalauting this paper.

\section{References}

Andronov, A. A. and Trakhtengerts, V. Yu.: Kinetic instability of the Earth's outer radiation belt, Geomagnetism and Aeronomy, 4, 233-242, 1964.

Bespalov, P. A. and Trakhtengerts, V. Yu.: Alfvén Masers. Preprint of the Institute of Applied Physics, Academy of Sciences of the USSR, 1986.

Brinca, A. L.: On the stability of Obliquely Propagating Whistlers, J. Geophys. Res., 77 (19), 3495-3507, 1972.

Burtis, W. J. and Helliwell, R. A.: Magnetospheric chorus: Occurrence patterens and normalized frequency, Planet. Space Sci., 24, 10007-10 024, 1976. 
Cairo, L. and F. Lefeuvre: Localization of sources of ELF/VLF hiss observed in the magnetosphere: Three-dimensional ray tracing, J. Geophys. Res., 91, 4352-4364, 1986.

Chum, J., Jiřiček, F., Smilauer, J., and Shklyar, D.: Magion 5 observations of chorus-like emissions and their propagation features as inferred from ray-tracing simulation, Ann. Geophys., 21, 22932302, 2003,

SRef-ID: 1432-0576/ag/2003-21-2293.

Gendrin, R.: Le guidage des whistlers par le champ magnetique, Planet. Space Sci., 5, 274-282, 1961.

Green, J. L., Scott, B., Garcia, L., Taylor, W. W. L., Fung, S. F., and Reinisch B. W.: On the origin of whistler mode radiation in the plasmasphere, J. Geophys. Res., 110(A03201), doi:10.1029/2004JA010495, 2005.

Gurnett, D. A. and Burns, T. B.: The low-frequency cutoff of ELF emissions, J. Geophys. Res., 73, 7437-7445, 1968.

Helliwell, R. A.: A theory of discrete emissions from the magnetosphere, J. Geophys. Res., 72, 4773-4790, 1967.

Helliwell, R. A.: Low-frequency waves in the magnetosphere, Rev. Geophys., 7, 281-303, 1969.

Kennel, C. F. and Petschek, H. E.: Limit on stably trapped particle fluxes, J. Geophys. Res., 71, 1-28, 1966.

Lauben, D. S., Inan U. S., and Bell T. F.: Source characteristics of ELF/VLF chorus, J. Geophys. Res., 107(A12), 1429, doi:10.1029/2000JA003019, 2002.

LeDocq, M. J., Gurnett, D. A., and Hospodarsky, G. B.: Chorus source locations from VLF Poynting flux measurements with the Polar spacecraft, Geophys. Res. Lett., 25, 4063-4066, 1998.

Nagano, I., Yagitani, S., Kojima, H., and Matsumoto, H.: Analysis of Wave Normal and Poynting vectors of Chorus emissions Observed by GEOTAIL, J. Geomag. Geoelectr., 48, 299-307, 1996.

Nunn, D., Omura, Y., Matsumoto, H., Nagano I., and Yagitani S.: The numerical simulation of VLF chorus and discrete emissions observed on Geotail satellite using a Vlasov code, J. Geophys. Res., 102, 27 083-27 097, 1997.

Nunn, D.: Vlasov hybrid simulation-an efficient and stable algorithm for the numerical simulation of collision-free plasma, Transparent Theory and statistical Physics, Proceedings of the Vlasovia Conference, Nancy, France, December 2003, 2004.

Parrot, M., Santolík, O., Cornilleau-Wehrlin, N., Maksimovic, M., and Harvey, C.: Magnetospherically reflected chorus waves revealed by ray tracing with CLUSTER data, Ann. Geophys., 21, 1111-1120, 2003,

SRef-ID: 1432-0576/ag/2003-21-1111.
Parrot, M., Santolík, O., Gurnett, D. A., Pickett, J. S., and Cornilleau-Wehrlin, N.: Characteristics of magnetospherically reflected chorus waves observed by CLUSTER, Ann. Geophys., 22, 2597-2606, 2004,

SRef-ID: 1432-0576/ag/2004-22-2597.

Santolík, O. and Parrot, M.: Case studies on wave propagation and polarization of ELF emissions observed by Freja around the local proton gyro-frequency, J. Geophys. Res., 104, 2459-2475, 1999.

Santolík, O. and Parrot, M.: Application of wave distribution function methods to an ELF hiss event at high latitudes, J. Geophys. Res., 105, 18 885-18 894, 2000.

Santolík O. and Gurnett, D. A.: Transverse dimensions of chorus in the source region, Geophys. Res. Lett, 30, 2, 1031, doi:10.1029/2002GL016178, 2003.

Santolík, O., Gurnett, D. A., and Pickett, J. S.: Multipoint investigation of the source region of storm-time chorus, Ann. Geophys., 22, 2555-2563, 2004,

SRef-ID: 1432-0576/ag/2004-22-2555.

Santolík, O., Gurnett, D. A., Pickett, J. S., Parrot, M., and Cornilleau-Wehrlin, N.: Central position of the source region of storm-time chorus, Planetary and Space Sci. 53, 299-305, 2005.

Shklyar, D. R. and Jiřiček, F.: Simulation of nonducted whistler spectrograms observed aboard the Magion 4 and 5 satellites, J. Atmos. S.-P., 62, 347-370, 2000.

Shklyar, D., Chum, J., and Jiřiček, F.: Characteristic properties of $\mathrm{Nu}$ whistlers as inferred from observations and numerical modelling, Ann. Geophys., 22, 3589-3606, 2004,

SRef-ID: 1432-0576/ag/2004-22-3589.

Stix, T. H.: Waves in Plasmas, Am. Inst. of Phys., New York, 1992.

Storey, L. R. O.: An investigation of whistling atmospherics, Phil. Trans. Roy. Soc. London, 246, 113-141, 1953.

Storey, L. R. O, Lefeuvre, F., Parrot, M., Cairo, L., and Anderson, R. R.: Initial survey of the wave distribution functions for plasmaspheric hiss observed by ISEE 1, J. Geophys. Res., 96, 19469-19489, 1991.

Thrakhtengerts, V. Y.: Magnetosphere cyclotron maser: backward wave oscillator generation regime, J. Geophys. Res., 100, 17 205-17 210, 1995.

Thrakhtengerts, V. Y.: A generation mechanism for chorus emission, Ann. Geophys., 17, 95-100, 1999,

SRef-ID: 1432-0576/ag/1999-17-95.

Tsurutani, B. T. and Smith, E. J.: Postmidnight chorus: a substorm phenomenon, J. Geophys. Res., 79, 118-127, 1974. 